\title{
Insights Arising from Gene Expression Profiling in Amyotrophic Lateral Sclerosis
}

\author{
Johnathan Cooper-Knock, Joanna J. Bury, Laura Ferraiuolo, \\ Emily F. Goodall, Pamela J. Shaw and Janine Kirby \\ Sheffield Institute for Translational Neuroscience, University of Sheffield, Sheffield, \\ United Kingdom
}

\section{Introduction}

Gene expression profiling has been used extensively in multiple fields of research to compare disease and control samples, to investigate mechanisms of disease, to identify subtypes within a heterogeneous clinical phenotype and to establish responses to a specific treatment. In addition, it can also be used to determine the function of a gene, to monitor changes over time, or for discovering cell specific expression or responses.

This chapter will initially explain the principles of gene expression profiling, before discussing the sources of samples, along with their advantages and limitations, and will then summarise the research to date regarding the application of gene expression profiling to amyotrophic lateral sclerosis (ALS).

\section{What is gene expression profiling?}

The primary application of gene expression profiling is to use a glass slide containing oligonucleotides or cDNA sequences, termed a microarray, to quantify the amount of individual RNA transcripts that are present in a particular cell or tissue. RNA is extracted, fluorescently labelled and then hybridized to the microarray. A linear amplification step can also be performed prior to labelling to ensure sufficient RNA is generated from low yields of RNA extracted from small sample sizes. The quantity of labelled RNA binding to each oligonucleotide or cDNA sequence on the microarray determines the intensity of fluorescence at that location and thus allows quantification of the RNA transcripts in the initial sample (Stoughton 2005).

Comparison of the expression of transcripts between sample groups allows identification of transcripts which are differentially expressed between the groups. The large number of genes simultaneously interrogated by a microarray means that the resulting data are amenable to study the expression of inter-related clusters of genes such as those involved in known functional categories or specific pathways. Changes in expression level of a particular gene do not take into account regulation at the level of translation. However, the functional impact of a differentially expressed gene can be subsequently established by assaying the encoded protein expression or function. 
The advent of next generation sequencing, and specifically the sequencing of all RNA molecules in a quantitative manner, has recently become an alternative, though expensive method for measuring levels of gene expression. However, this has the potential to add further knowledge and value to the application of gene expression profiling to disease.

\section{Uses of gene expression profiling}

Quantification of the transcriptome has been a useful mechanism for both discovering and defining mechanisms of pathogenesis in ALS (Cox et al 2010; Ferraiuolo et al 2007; Kirby et al 2005; Kirby et al 2011). In particular lists of differentially expressed genes can be usefully converted to functional 'themes' by an enrichment analysis (Hosack et al 2003). Various categorisations exist, including the gene ontology (GO) and Kyoto encyclopaedia of genes and genomes (KEGG), which classify genes according to molecular function, biological process, cellular component or a known biological pathway.

A frequent application of gene expression profiling has been the development of putative biomarkers via a supervised classification approach (Booij et al 2011; Nagasaka et al 2005; Scherzer et al 2007). The large number of targets quantified simultaneously by gene expression profiling is essential for biomarker discovery, as it allows an unbiased survey of the most informative RNA transcripts. A reliable biomarker(s) for defining pathogenesis and prognosis in ALS has yet to be established, though gene expression profiling is one of the methodologies currently being used to establish an ALS biomarker(s).

\section{Sources of material for studying ALS}

In ALS, the vulnerable cells are the motor neurones, located in the motor cortex, brainstem and spinal cord. Since motor neurones cannot be sampled during life, model systems, such as neuronal-like cells in culture and animals carrying mutant transgenes, have been used to study the neurodegenerative process. In addition to sampling post-mortem material from patients, peripheral tissue from living patients (and neurologically normal controls) has been used as a source material for applying gene expression profiling to ALS.

\subsection{Cellular models of ALS}

Neuronal cell lines, both human (e.g. SH-SY5Y) and rodent (e.g. PC12), have been used as a model to investigate mechanisms of neurodegeneration. However, one of the most widely used cellular models for examining the molecular pathophysiology underpinning the neurodegenerative disease process in ALS is the mouse spinal cord/neuroblastoma cell line NSC-34 (Cashman et al 1992). Immortalized NSC-34 cells recapitulate many of the characteristics of motor neurones whilst maintaining their ability to proliferate in culture; thus providing a continual resource of motor neurone-like cells (Cashman et al 1992; Durham et al 1993). In particular, they have proved a robust model of mutant copper zinc superoxide dismutase-1 (SOD1) associated familial ALS (FALS), as they can be transfected with vectors carrying normal or mutant forms of the human SOD1 gene (Durham et al 1997; Menzies et al 2002a; Menzies et al 2002b). Cellular models of TDP-43-related ALS, caused by mutation of the TAR DNA binding protein gene (TARDBP) are being generated (Duan et al 2010; Igaz et al 2009), though sustained over-expression of the wild-type and mutant proteins is proving problematic due to the toxicity of both the wild-type and mutant overexpressed proteins and the tight auto-regulation of TDP-43 (Budini \& Buratti 2011). 
Gene expression profiling of cell lines transfected with ALS associated genes provides a genetically homogenous cell population uncontaminated by non-neuronal astrocytes and other types of glial cells, which are present in the central nervous system (CNS). Furthermore, environmental conditions can easily be manipulated and tightly controlled in vitro so as to reduce the impact of external confounding factors on gene expression. The limitations of this type of model system include the fact that NSC-34 cell lines (or other neuronal cell lines) are continually dividing cells, rather than post-mitotic cells and they are unable to mirror the effects of cellular interactions that occur between the different cell populations in situ (Kirby et al 2011).

Primary neuronal and astrocytic cells can be isolated from embryonic mice and short-term cultures generated for microarray analysis. These cells more closely mirror those present in the CNS, though the primary neuronal cultures, as they are post-mitotic cells, have a limited lifespan of 7-10 days. In contrast, cultured primary astrocytes are able to proliferate in culture. Whilst co-cultures or separated co-cultures allow a degree of interaction between the two cell types, these types of mixed cultures have not yet been used for microarray analysis in ALS.

\subsection{Animal models of ALS}

Transgenic mice expressing mutant forms of ALS-related genes provide a source of RNA for microarray analysis. For investigating the mechanisms of SOD1-related neurodegeneration, mice over-expressing the human p.G93A or mouse p.G86R mutant forms of the SOD1 protein (SOD1G93A or SOD1G86R) have been used as they develop an age dependent neuromuscular condition; the motor function symptoms and histopathological features have been extensively characterised and resemble those observed in both SOD1-related ALS and classical ALS patients (Gurney et al 1994; Ripps et al 1995). In contrast, over-expression of wild-type human SOD1 (SOD1WT) does not produce an overt motor phenotype, supporting a toxic gain of function by the mutant SOD1 protein as the mechanism by which the mutant proteins cause cell death. In contrast, mouse models over-expressing either wild-type or mutant TDP-43 show a neurodegenerative phenotype (Igaz et al 2011; Stallings et al 2010).

One of the major advantages of using animal models for microarray analysis is the ability to examine animals at different ages in order to investigate the progression of disease, an approach that is unattainable in human post-mortem tissue. Valuable insights regarding onset of disease can be established in pre-symptomatic and early symptomatic disease stages since these represent time points at which the identification of key novel targets for therapeutic intervention could be best placed to rescue vulnerable neuronal cell populations before the development of irreversible neuronal injury (Ferraiuolo et al 2007). In addition, sampling of specific cell types from the CNS allow gene expression changes to be identified which include the effects of interactions with neighbouring cells.

Backcrossing of the SOD1WT and SOD1G93A mice with C57B16 mice has led to the formation of SOD1WT and SOD1G93A mice on a homogeneous background (Ferraiuolo et al 2007). The use of these mice for microarray analysis and the use of non-transgenic littermates as controls have proven effective in reducing inter-individual genetic variability to ensure the generation of consistent and reliable gene expression data.

\subsection{Human post-mortem material}

Human post-mortem brain and spinal cord specimens derived from clinically and pathologically confirmed cases of ALS can be used in comparisons with age, gender and 
ethnically matched neurologically normal controls and are a pivotal source of RNA for gene expression profiling in ALS. Brain and spinal cord represent the tissues that are most susceptible to the underlying neurodegenerative disease process. However, there are a number of limitations that should be taken into account when using this tissue. First and foremost, the transcriptional changes that are detected are reflecting the terminal stage of disease, with the majority of vulnerable neuronal cells having already undergone cell death (Sharp et al 2006). Therefore, it can be difficult to distinguish whether the changes detected are due to the survival response in the remaining cells, initiation of a cell death pathway (if the cells are beginning to die), or whether the changes are present in response to a pathogenic trigger (Lederer et al 2007). Secondly, samples, and particularly control material, are difficult to obtain and often in short supply. This restricts the sample sizes that can be used, which has a negative impact upon the statistical power of such studies. Considerable heterogeneity exists between individuals in disease and control groups and gene expression changes may also be influenced by post-mortem interval, variability in brain $\mathrm{pH}$, degree of neuroinflammation and sample type including white versus grey matter or cortical motor neurones versus spinal motor neurones. Furthermore, ex vivo RNA degradation, particularly during sample preparation, should also be taken into consideration (Maes et al 2007).

RNA extraction protocols are faster and more straightforward for whole tissue homogenates. However, the inclusion of a heterogeneous mixture of cell populations may in effect blur the distinctive profile emanating from the neuronal cells that are most affected in ALS (Kirby et al 2011). In comparing the gene expression profiles generated from diseased tissue to that of healthy controls there is also the added issue of a shift in the proportion of different cell populations within the sample as neurodegeneration is characterised by the loss of motor neurones and the active proliferation of non-neuronal microglia and macrophages (Dangond et al 2004). Laser capture microdissection (LCM) may be a more expensive and laborious technique but is beneficial in studying a neuronal enriched cell population (Jiang et al 2005). It also is noteworthy that in comparison to primary cultures these cells have had the benefit of being embedded within their natural environment where physiologically relevant cross talk has taken place with neighbouring cells and tissues (Ferraiuolo et al 2007).

\subsection{Human peripheral tissue samples}

Peripheral tissue such as whole venous blood, cultured skin fibroblasts and muscle biopsy material offer an attractive and readily accessible resource for microarray analysis in ALS. Samples can be collected longitudinally, particularly as the collection of blood is relatively non-invasive and sampling techniques can be standardized across research centres (Highley et al 2011; Saris et al 2009; Shtilbans et al 2011; Tsuang et al 2005).

Blood is classified as a fluid connective tissue composed of plasma (55\%), erythrocytes $(43 \%)$, leukocytes $(0.5 \%)$ and platelets $(1.5 \%)$ which continuously permeates and interacts with every other tissue and organ of the mammalian body. It is in a permanent state of renewal and is known to play a pivotal role in physiological homeostasis, cellular immunity and inflammation (Mohr \& Liew 2007). Since $80 \%$ of the genes routinely expressed within the CNS have also been detectable in circulating blood cells it is anticipated that there are quantifiable changes in the levels of these gene transcripts which have the potential to act as a sentinel of disease (Liew et al 2006). Evidence suggests that the shear abundance of endogenous alpha and beta globin messenger RNA (mRNA), which constitutes up to $70 \%$ of 
erythrocytic mRNA transcripts, masks the expression of less abundant genes of potential biological significance as a result of their high signal intensity on the microarray (Wright et al 2008). Thus, strategies have been developed and evaluated to remove these transcripts present in the RNA samples from whole blood (Liu et al 2006). Alternatively, fractionation of peripheral blood mononuclear cells (PBMCs) can also be used though the additional processing steps involved in isolating the PBMCs can introduce spurious artifactual alterations in gene expression that are not attributable to the disease (Whitney et al 2003).

Fibroblasts are not known to have any direct involvement in ALS, though they provide a model with the genetic background of the individual and have the added value of being a source for the generation of induced pluripotent stem cells (iPS) or for the direct manipulation into motor neuronal cells (Dimos et al 2008; Son et al 2011). In addition, fibroblasts have been shown to reflect changes in patients with neurodegenerative disease (Aguirre et al 1998; Hoepken et al 2008; Mortiboys et al 2008) and their gene expression profiles distinguish pre-symptomatic individuals from those of controls (Nagasaka et al 2005). In contrast to fibroblasts, skeletal muscle is severely affected by the disease. Whilst muscle biopsies are the most invasive sample to collect, they are a useful source for gene expression profiling, as they provide a window into the mechanisms involved in the neuromuscular degeneration that occur in ALS during life (Dadon-Nachum et al 2011; Dupuis \& Loeffler 2009).

\section{Results from use of cellular models of ALS}

Gene expression profiling of the widely used NSC34 cellular model of SOD1-related FALS identified a marked degree of transcriptional repression in the presence of the SOD1G93A mutation (Kirby et al 2005). These repressed genes included a group of antioxidant response (ARE) genes or "programmed cell life" genes that are regulated by the Nrf2 transcription factor (Figure 1). Reduced expression of Nrf2 and selected downstream targets was seen at both the RNA and protein levels in the cellular model and NRF2 dysregulation was also demonstrated in isolated motor neurones from SOD1-related ALS cases. Subsequent work by Sarlette and colleagues has shown that NRF2 transcription and translation is also decreased in SALS cases (Sarlette et al 2008). Most recently, it has been demonstrated that activation of Nrf2 in NSC34 SOD1-related ALS cell models, primary motor neurone and astrocyte co-cultures and in the SOD1G93A mouse model all improve neuronal survival (Neymotin et al 2011; Vargas et al 2008a).

Primary astrocyte cultures prepared from mutant SOD1 rodent models have been shown to increase motor neuronal death in co-cultures (Nagai et al 2007; Vargas et al 2006). To investigate this effect, gene expression profiling was performed on RNA isolated from astrocyte cultures generated from SOD1G93A rats (and litter mate controls). Perhaps surprisingly, there were limited differences in the transcriptional profiles of transgenic and non-transgenic astrocytes (Vargas et al 2008b). However, of the two genes most dysregulated, regulator of differentiation (Rod1) and decorin $(D c n)$, both showed consistent changes in asymptomatic and early symptomatic rats, implicating components of RNA processing and the extracellular matrix as contributors early in the disease process (Figure 1).

Primary neuronal cultures originating from SOD1G93A mice and subjected to oxidative stress $\left(\mathrm{H}_{2} \mathrm{O}_{2}\right)$ or excitotoxicity (NMDA), demonstrated a greater level of cell death, compared to non-transgenic cultures (Boutahar et al 2011). Microarray analysis detected cytoskeletal remodelling and vesicular transport related genes as increased in response to oxidative 
stress, whilst genes involved in the ubiquitin-proteasome system and cytokines were increased following excitotoxicity (Figure 1). Several of these pathways have already been implicated as playing a pathogenic role in ALS and add further support to the idea that the proposed disease mechanisms are mutually compatible.

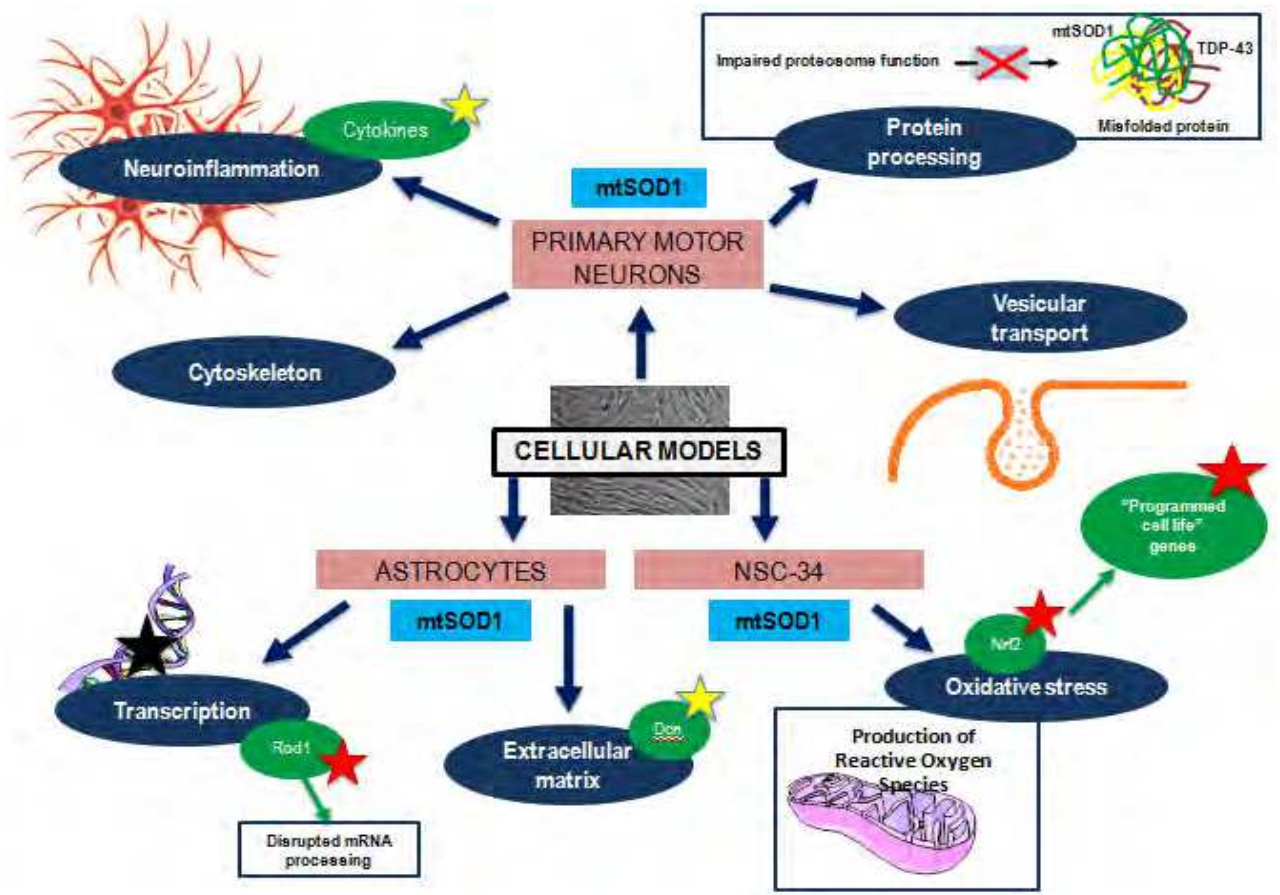

Fig. 1. Summary of prominent pathways arising from GEP of Cellular Models. Important changes in the transcriptome have been highlighted by green labels; yellow stars indicate up-regulation, red stars indicate down-regulation. Blue squares outline functional consequences of changes in the transcriptome. Further details are discussed in the text.

\section{Results from use of animal models of ALS}

\subsection{Gene expression profiling of mixed cell type CNS samples}

Microarray analysis of whole spinal cord homogenates from ALS mouse models have been performed by several research groups in order to obtain a global view of changes in the CNS prior to and during disease. Results from SOD1G93A mice have shown that inflammation, apoptosis and adaptive responses to metal ion dysregulation are the main pathways activated in both pre-symptomatic mice and during the disease process (Olsen et al 2001; Yoshihara et al 2002) (Figure 2). Analysis of the SOD1L126delTT transgenic mouse, which results in a truncated SOD1 protein, also showed pre-symptomatic changes related to the reactive gliosis which is occurring in the spinal cord (Fukada et al 2007).

Recently, transgenic mice carrying mutant TDP-43 have also contributed to better understanding the different mechanisms involved in ALS. Transgenic mice induced to 
express human TDP-43 without the nuclear localization signal (hTDP43-delNLS) developed signs of motor spasticity, neurone loss in forebrain regions and corticospinal tract degeneration (Igaz et al 2011). Microarray analysis of hTDP43-delNLS expression in the cortex of mutant mice, following 2 weeks induction of the mutant protein, detected dramatic changes in gene expression, with the most enriched pathway being chromatin assembly (Figure 2). Interestingly, after only 2 weeks of hTDP43-delNLS induction, markers of inflammation and neuronal loss were unchanged.

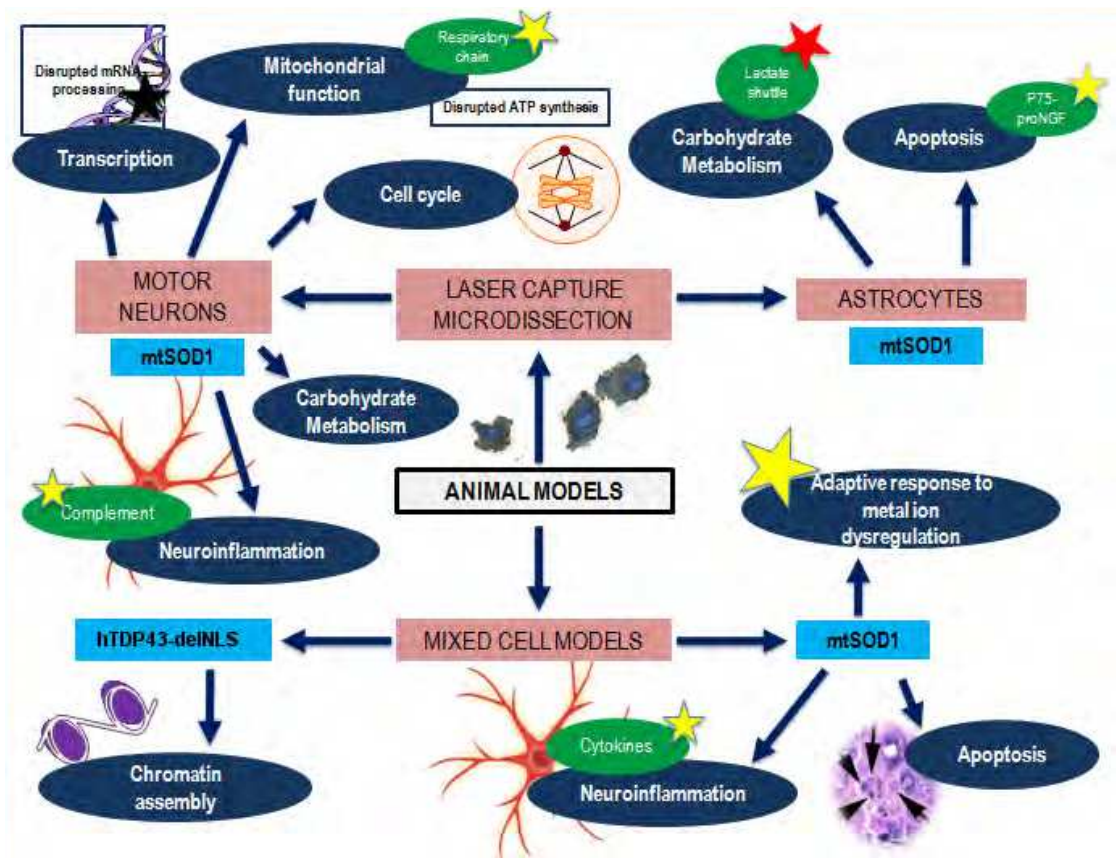

Fig. 2. Summary of prominent pathways arising from GEP of Animal Models. Important changes in the transcriptome have been highlighted by green labels; yellow stars indicate up-regulation, red stars indicate down-regulation. Blue squares outline functional consequences of changes in the transcriptome. Further details are discussed in the text.

Although these studies have greatly contributed to present knowledge on the transcriptional changes occurring in ALS, the analysis of a mixed cell population within the CNS has several disadvantages. This kind of approach does not identify which cell population is responsible for the transcriptional changes observed and only detects those transcripts most highly differentially expressed, with subtle but potential pivotal gene expression changes masked as well as changes in genes differentially expressed in one cell type, but not in others.

\subsection{Gene expression profiling of laser capture microdissection isolated cell types}

In order to overcome the limitations of using mixed cell population samples, dissection of single cells from complex tissues using LCM has been applied to identify the contribution of different cell types to the degenerative process occurring in ALS. 
Several studies have determined the changes in gene expression occurring in motor neurones isolated from the spinal cord of mutant SOD1G93A mice at different stages during the disease; from the pre-symptomatic stage to paralysis (Ferraiuolo et al 2007; Perrin et al 2005). The first report described transcriptional analysis of motor neurones isolated from SOD1G93A mice bred on a mixed background and no differentially expressed genes were detected in the pre-symptomatic mice (Perrin et al 2005). However, in contrast to the whole tissue homogenates, motor neurones did not show activation of apoptotic genes, suggesting that cell death signals derive from other cell types in the spinal cord (Figure 2).

In the second publication, microarray analysis was carried out on SOD1G93A mice bred on a homogeneous background; this enabled important changes in the motor neurones at the pre-symptomatic stage of disease, mainly involved in carbohydrate metabolism and transcription, to be detected (Ferraiuolo et al 2007). The upregulation of transcripts encoding proteins involved in the energy production pathway, i.e. tricarboxylic acid cycle and respiratory chain, suggested that motor neurones were trying to compensate for their increased energy needs in response to ongoing stress. At the late stage of disease, increased expression of transcripts involved in reactivation of the cell cycle (as an alternative pathway of cell death), and complement activation (a mechanism through which motor neurones can attract cells from the immune system), and down-regulation of transcription-related genes were identified (Figure 2).

To complement the gene expression profiling of motor neurones, astrocytes isolated from SOD1G93A mice at the pre-symptomatic stage of disease were isolated and used for microarray analysis (Ferraiuolo et al 2011a). This enabled the cross-talk between the motor neurones and astrocytes at this very early time point to be interrogated. Interestingly, astrocytes displayed a marked impairment of carbohydrate metabolism (Figure 2). Comparing the expression profiles of the two cell types from the same SOD1G93A mice highlighted that the metabolic impairment observed in motor neurones could derive from the lack of provision of substrates, i.e. lactate, from the astrocytes, and led to the conclusion that the lactate shuttle (the mechanism through which motor neurones and astrocytes combine metabolism and signalling through lactate and glutamate), is impaired. In addition, the activation of an important neuronal cell death pathway involving p75 and its ligand pro nerve growth factor (proNGF) was established. Gene expression profiling of SOD1G93A astrocytes demonstrated that these cells expressed high levels of $N g f$, while the motor neurones over-expressed the $\mathrm{p} 75$ receptor. In vitro data confirmed the dysregulation of both pathways and preliminary data from human ALS biosamples supported these findings from the murine model.

\subsection{Gene expression profiling of peripheral tissue from mouse models}

Microarray technology has also been applied to peripheral tissues from the SOD1G86R mouse model (Gonzalez de Aguilar et al 2008). Profiling of the skeletal muscles revealed that the major expression changes happen at onset of disease, when muscles are activating pathways involved in detoxification and regeneration, but also cell death and tissue degradation. These findings revealed that while motor neurones are degenerating, muscles are undergoing major remodelling trying to compensate for muscle damage with new myogenesis. Whilst over-expression of transcripts such as cyclin-dependent kinase inhibitor-1A (Cdkn1a) and growth arrest-and DNA damage-inducible gene-45 (Gadd45) could be mediating apoptosis of myofibres resulting in muscle atrophy, increased 
expression B cell translocation gene-1 (Btg1), growth differentiation factor-5 (Gdf5) and myogenic factor-6 (Myf6) are potent activators of new fibre formation.

\section{Results from use of human post-mortem material}

\subsection{Gene expression profiling of mixed cell type CNS samples}

Multiple studies have utilised gene expression profiling of post mortem mixed cell samples from ALS patients and controls; these have either focused on using samples from the motor cortex (Lederer et al 2007; Wang et al 2006) or from the spinal cord (Dangond et al 2004; Malaspina et al 2001; Offen et al 2009). The majority used sporadic ALS (SALS) cases, though Dangond et al also sampled two FALS cases, one of which carried an SOD1 mutation (Dangond et al 2004). Despite the different tissues profiled and the different platforms utilised, the studies showed some consistent results: All of the studies recorded altered gene expression related to inflammation and Malaspina and colleagues detected an increase in glial fibrillary acidic protein (GFAP), indicating active astrogliosis (Figure 3). In addition, a number of the studies discovered differential expression related to cytoskeleton function, protein processing and the antioxidant response, in agreement with other lines of research in ALS (Ferraiuolo et al 2011b).

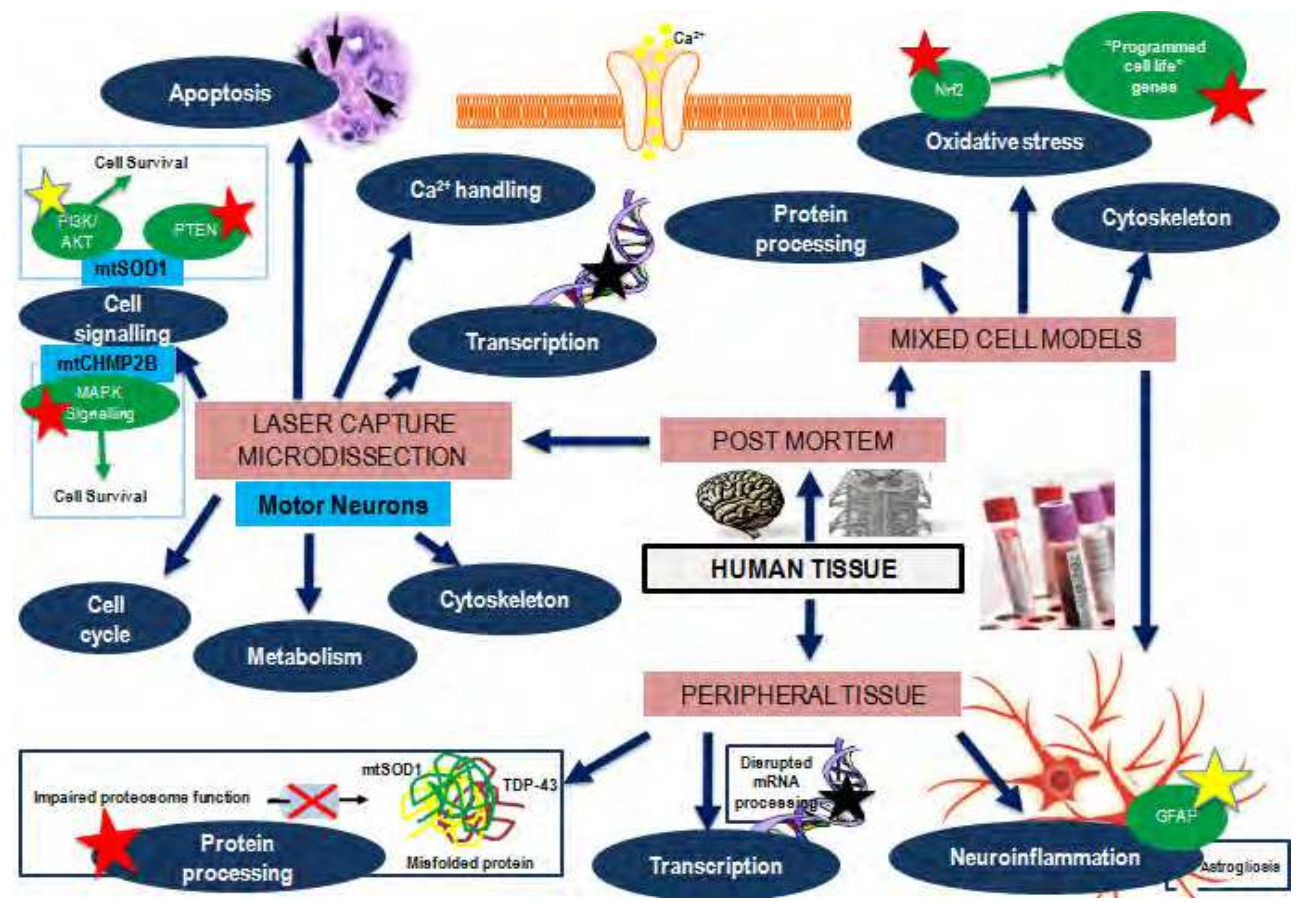

Fig. 3. Summary of prominent pathways arising from GEP of Human Tissue. Important changes in the transcriptome have been highlighted by green labels; yellow stars indicate up-regulation, red stars indicate down-regulation. Blue squares outline functional consequences of changes in the transcriptome. Further details can be found in the text. 


\subsection{Gene expression profiling of laser capture microdissection isolated cell types}

As with the mouse models, in order to determine those genes differentially expressed in the vulnerable cell type, LCM has been used in post-mortem material to isolate the motor neurones from the spinal cord.

Motor neurones isolated from SALS cases and neurologically normal controls were shown to have distinct gene expression profiles compared to those generated from ventral horn homogenates, particularly with respect to those genes down-regulated in motor neurones (Jiang et al 2005). The motor neurones showed differential expression of genes associated with the cytoskeleton and evidence of decreased transcription, whilst cell death-associated genes and those involved in cell signalling were increased (Figure 3). In addition, cell cycle related genes were also reported as dysregulated, supporting the theory that inappropriate activation of the cell cycle in these post-mitotic cells leads to cell death. A follow-up study demonstrated that expression of several of these genes also correlated with pathological markers, such as phosphorylated neurofilament and ubiquitinated protein accumulations as well as motor neurone loss (Jiang et al 2007).

Gene expression profiling of isolated motor neurones has also been performed on ALS cases which carry genetic mutations in the SOD1 and chromatin modifying protein $2 \mathrm{~B}(C H M P 2 B)$ genes (Cox et al 2010; Kirby et al 2011). Motor neurones from SOD1-related cases of ALS showed increases in genes in the protein kinase $B /$ phosphatidylinositol-3 kinase (AKT/PI3K) cell survival pathway, with concomitant decreases in negative regulators such as phophastase and tensin homologue (PTEN) (Kirby et al 2011) (Figure 3). Further work demonstrated that inhibition of PTEN led to increased activation of the AKT/PI3K pathway and increased neuronal survival in cell models including primary motor neurone cultures. Thus, activation of the AKT/PI3K pathway was proposed as a candidate for future therapeutic strategies.

The transcriptional profiles from motor neurones isolated from the CHMP2B-related ALS cases were distinct from those in SOD1-related cases (Cox et al 2010). These motor neurones showed dysregulation of genes involved in the classical and p38 MAPK signalling pathways, gene changes predicted to reflect reduced autophagy and repression of translation (Figure 3). The functional implication of $C H M P 2 B$ mutations on cellular mechanisms was demonstrated by the presence of large cytoplasmic vacuoles and impairment of autophagy in a cellular model transfected with mutant $C H M P 2 B$, consistent with the microarray findings (Cox et al 2010). Interestingly, differential expression of genes encoding proteins responsible for calcium handling and cell cycle genes, as well as those genes involved in transcription, signalling and metabolism, was detected in both genetic subtypes.

\section{Results from use of human peripheral tissue}

Gene expression profiling has been conducted on blood and fibroblasts from ALS patients (Highley et al 2011; Saris et al 2009; Zhang et al 2011). Microarray analysis of SALS and control blood samples was followed by hierarchical clustering of all genes found to be significantly expressed in all samples after normalisation (Saris et al 2009). The method identified five clusters; two of which were able to differentiate between ALS and control samples. These clusters were replicated in a further two cohorts of patients and controls, demonstrating that such an analysis, which takes into account the interdependence of gene expression, is a means of reducing the false negative rate when subsequently detecting 
differential gene expression. This work also provided evidence that peripheral blood is a valuable medium for studying ALS. In addition, there was a correlation with the CNS tissue studies, as the blood in ALS patients showed a decrease in genes associated with protein processing and RNA post-transcriptional modification, as well as increases in genes associated with inflammation.

A more recent study performed gene expression profiling on peripheral blood mononuclear cells from patients with SALS (Zhang et al 2011). These cells showed an up-regulation of genes associated with immune activation in response to lipopolysaccharide (LPS), which correlated with an elevation of plasma LPS. Unfortunately, no correlation between expression of these genes and disease progression was provided.

Transcriptional profiles of fibroblasts from patients with SALS, FALS and controls have been shown to be informative in distinguishing the different genetic variants from each other, as well as from SALS patients, by their gene expression profiles and specifically the level of alternative splicing (Highley et al 2011). The FALS samples were derived from patients with mutations in SOD1 and TARDBP. Using microarrays which interrogate every exon of every gene, it was demonstrated that there was a significant amount of aberrant splicing in the samples with a TARDBP mutation which was replicated to a lesser degree in the SALS samples and virtually absent in the samples with a SOD1 mutation (Figure 3). In support of this, other work has identified aberrant splicing in cell and animal models with depletion of TDP-43 (Polymenidou et al 2011; Tollervey et al 2011).

\section{The future of gene expression profiling in ALS}

\subsection{RNA sequencing (RNA-seq)}

The advent of next generation sequencing has evolved to enable quantitative parallel sequencing of RNA transcripts from isolated cells and tissues. There are a number of advantages of next generation RNA sequencing over the microarray platform which in general extend from the fact that there is no reliance on the pre-designed probes which are present on the microarrays. In contrast, the methodology aims to sequence every base of every transcript of RNA. This leads to a better detection rate of known transcripts and splicing events and the detection of RNA transcripts (both coding and non-coding) which have not previously been described and therefore have no specific probe (Sultan et al 2008). As there is no reliance on probes, the problem of cross hybridisation is avoided. In addition, because each base in each transcript is sequenced, as well as providing information about expression level and alternative splicing, the sequencing also provides information about sequence variability within the RNA (Wang et al 2009). The biggest challenge to RNA sequencing, however, is the analysis of the large amounts of data produced which is substantially more than the read out of even the most comprehensive microarray. Not least among these challenges is the problem of mapping the RNA sequences to the genome, as in contrast to DNA sequencing, the absence of introns can lead to substantial difficulties (Sutherland et al 2011).

\subsection{Role of microRNAs in ALS}

This chapter has focused on the mRNA that is translated into protein. However, although $90 \%$ of eukaryotic genomic DNA is transcribed, only $1-2 \%$ actually encodes protein. The vast majority of transcribed material is comprised of non-coding RNA (ncRNA) and there is 
increasing evidence to support functional roles for at least a subset of these transcripts (Kaikkonen et al 2011). There are broadly two types of ncRNA, infrastructural (including transfer RNA and small nuclear RNA) and regulatory RNA (including microRNA, Piwiinteracting RNA and small interfering RNA). The function of ncRNAs remains largely unknown. However, research into microRNA (miRNA) has led the field in recent years. miRNAs are a class of small, ncRNA molecules predicted to post-transcriptionally regulate at least one third of human genes (Lewis et al 2005). Each miRNA can potentially target hundreds of genes and play key regulatory roles in a diverse range of pathways including development, differentiation and pathological processes such as neurodegeneration (Enciu et al 2011). The study of miRNA in ALS is at a very early stage. However, given the proposed role for TDP-43 in miRNA biogenesis and the recent discovery of a beneficial effect of miRNA-206 in the mutant SOD1 mouse model, this will be an interesting area of investigation for the future (Buratti et al 2010; Williams et al 2009)

\subsection{Biomarkers in ALS}

There is no diagnostic test for ALS, so diagnosis currently relies upon clinical assessment involving the exclusion of "ALS-mimic" syndromes (such as multifocal motor neuropathy and cervical radiculomyelopathy), causing an average delay of one year from symptom onset to a confirmed diagnosis (Silani et al 2011; Zoccolella et al 2006). In such a rapidly progressive disease this delay is a significant obstacle to potential neuroprotective therapies. ALS is clinically heterogeneous, with multiple subtypes associated with different survival times and symptoms making prognosis challenging. This heterogeneity is also a confounding factor for clinical trials as patient phenotype will impact upon survival data and may influence responses to therapeutic intervention, with some subtypes more responsive to therapy than others (Turner et al 2009). Robust biomarkers would therefore be valuable for the initial diagnosis of disease, the classification of various subtypes, monitoring responses to therapeutic agents and tracking disease progression (Turner et al 2009). Gene expression profiling offers a useful tool for biomarker discovery allowing patient and control biofluids, such as blood and CSF, to be compared on a genome wide scale. These tools have already been employed to improve classification and diagnosis of multiple diseases including neurodegenerative conditions such as Huntington's disease and Parkinson's disease (Borovecki et al 2005; Scherzer et al 2007).

\section{Conclusion}

In conclusion, microarray analysis has been pivotal in understanding the transcriptional alterations occurring in response to genetic mutations associated with ALS and the sporadic disease (SALS). The cellular model has generated a therapeutic target and transcriptional activation of Nrf2 is currently being assessed in-vivo. Use of spinal cord and peripheral tissues from transgenic mouse models has provided a mechanism to look at the progression of the disease and specifically to identify early changes in the motor neurones and astrocytes. These dysregulated pathways provide future therapeutic targets. In addition, gene expression profiling has allowed crucial insights into the mechanisms affecting different areas of the motor system, with the combination of LCM and microarray technology able to discriminate changes in specific cell types and understand how these affect each other and contribute to disease progression. Finally, the use of human material 
has begun to allow the distinctions between genetic and sporadic disease to be differentiated as well as providing further candidates for therapeutic approaches. In the future, gene expression profiling of larger, consistently collected patient samples has the potential to generate robust and reliable prognostic and diagnostic biomarkers, which will ultimately be applicable for use in the clinic.

\section{Acknowledgments}

PJS and JK are funded by the European Community's Health Seventh Framework Programme (FP7/2007-2013) under grant agreement 259867 and by the Motor Neurone Disease Association (MNDA). EFG is also funded by the MNDA.

\section{References}

Aguirre T, Van Den Bosch L, Goetschalckx K, Tilkin P, Mathijs G, et al. 1998. Increased sensitivity of fibroblasts from amyotrophic lateral sclerosis patients to oxidative stress. Ann Neurol 43:452-7

Booij BB, Lindahl T, Wetterberg P, Skaane NV, Saebo S, et al. 2011. A gene expression pattern in blood for the early detection of Alzheimer's disease. J Alzheimers Dis 23:109-19

Borovecki F, Lovrecic L, Zhou J, Jeong H, Then F, et al. 2005. Genome-wide expression profiling of human blood reveals biomarkers for Huntington's disease. Proc Natl Acad Sci U S A 102:11023-8

Boutahar N, Wierinckx A, Camdessanche JP, Antoine JC, Reynaud E, et al. 2011. Differential effect of oxidative or excitotoxic stress on the transcriptional profile of amyotrophic lateral sclerosis-linked mutant SOD1 cultured neurons. J Neurosci Res 89:1439-50

Budini M, Buratti E. 2011. TDP-43 Autoregulation: Implications for Disease. J Mol Neurosci

Buratti E, De Conti L, Stuani C, Romano M, Baralle M, Baralle F. 2010. Nuclear factor TDP43 can affect selected microRNA levels. FEBS J 277:2268-81

Cashman NR, Durham HD, Blusztajn JK, Oda K, Tabira T, et al. 1992. Neuroblastoma x spinal cord (NSC) hybrid cell lines resemble developing motor neurons. Dev Dyn 194:209-21

Cox LE, Ferraiuolo L, Goodall EF, Heath PR, Higginbottom A, et al. 2010. Mutations in CHMP2B in lower motor neuron predominant amyotrophic lateral sclerosis (ALS). PLOS ONE 5:e9872

Dadon-Nachum M, Melamed E, Offen D. 2011. The "dying-back" phenomenon of motor neurons in ALS. J Mol Neurosci 43:470-7

Dangond F, Hwang D, Camelo S, Pasinelli P, Frosch MP, et al. 2004. Molecular signature of late-stage human ALS revealed by expression profiling of postmortem spinal cord gray matter. Physiol Genomics 16:229-39

Dimos JT, Rodolfa KT, Niakan KK, Weisenthal LM, Mitsumoto H, et al. 2008. Induced pluripotent stem cells generated from patients with ALS can be differentiated into motor neurons. Science 321:1218-21

Duan W, Li X, Shi J, Guo Y, Li Z, Li C. 2010. Mutant TAR DNA-binding protein-43 induces oxidative injury in motor neuron-like cell. Neuroscience 169:1621-9 
Dupuis L, Loeffler JP. 2009. Neuromuscular junction destruction during amyotrophic lateral sclerosis: insights from transgenic models. Curr Opin Pharmacol 9:341-6

Durham HD, Dahrouge S, Cashman NR. 1993. Evaluation of the spinal cord neuron X neuroblastoma hybrid cell line NSC-34 as a model for neurotoxicity testing. Neurotoxicology 14:387-95

Durham HD, Roy J, Dong L, Figlewicz DA. 1997. Aggregation of mutant Cu/Zn superoxide dismutase proteins in a culture model of ALS. J Neuropathol Exp Neurol 56:523-30

Enciu AM, Popescu BO, Gheorghisan-Galateanu A. 2011. MicroRNAs in brain development and degeneration. Mol Biol Rep 10.1007/s11033-011-0973-1:(Epub ahead of print)

Ferraiuolo L, Heath PR, Holden H, Kasher P, Kirby J, Shaw PJ. 2007. Microarray analysis of the cellular pathways involved in the adaptation to and progression of motor neuron injury in the SOD1 G93A mouse model of familial ALS. J Neurosci 27:920119

Ferraiuolo L, Higginbottom A, Heath P, Barber S, Greenald D, et al. 2011a. Dysregulation of astrocyte-motor neuron cross-talk in mutant SOD1 related amyotrophic lateral sclerosis. Brain (In Press)

Ferraiuolo L, Kirby J, Grierson AJ, Sendtner M, Shaw PJ. 2011b. Molecular and cellular pathways of motor neuron injury in amyotrophic lateral sclerosis. Nat Rev Neurology (In Press)

Fukada Y, Yasui K, Kitayama M, Doi K, Nakano T, et al. 2007. Gene expression analysis of the murine model of amyotrophic lateral sclerosis: studies of the Leu126delTT mutation in SOD1. Brain Res 1160:1-10

Gonzalez de Aguilar JL, Niederhauser-Wiederkehr C, Halter B, De Tapia M, Di Scala F, et al. 2008. Gene profiling of skeletal muscle in an amyotrophic lateral sclerosis mouse model. Physiol Genomics 32:207-18

Gurney ME, Pu H, Chiu AY, Dal Canto MC, Polchow CY, et al. 1994. Motor neuron degeneration in mice that express a human $\mathrm{Cu}, \mathrm{Zn}$ superoxide dismutase mutation. Science 264:1772-5

Highley JR, Hewamadduma C, Jansweijer JA, Kirby J, Heath PR, et al. 2011. Amyotrophic lateral sclerosis-causing TARDBP (TDP-43) mutations cause widespread dysregulation of mRNA splicing in cell lines from human patients. PLoS ONE (Under Review)

Hoepken HH, Gispert S, Azizov M, Klinkenberg M, Ricciardi F, et al. 2008. Parkinson patient fibroblasts show increased alpha-synuclein expression. Exp Neurol 212:30713

Hosack DA, Dennis G, Jr., Sherman BT, Lane HC, Lempicki RA. 2003. Identifying biological themes within lists of genes with EASE. Genome Biol 4:R70

Igaz LM, Kwong LK, Chen-Plotkin A, Winton MJ, Unger TL, et al. 2009. Expression of TDP43 C-terminal Fragments in Vitro Recapitulates Pathological Features of TDP-43 Proteinopathies. J Biol Chem 284:8516-24

Igaz LM, Kwong LK, Lee EB, Chen-Plotkin A, Swanson E, et al. 2011. Dysregulation of the ALS-associated gene TDP-43 leads to neuronal death and degeneration in mice. $J$ Clin Invest 121:726-38 
Jiang YM, Yamamoto M, Kobayashi Y, Yoshihara T, Liang Y, et al. 2005. Gene expression profile of spinal motor neurons in sporadic amyotrophic lateral sclerosis. Ann Neurol 57:236-51

Jiang YM, Yamamoto M, Tanaka F, Ishigaki S, Katsuno M, et al. 2007. Gene expressions specifically detected in motor neurons (dynactin 1, early growth response 3, acetylCoA transporter, death receptor 5, and cyclin C) differentially correlate to pathologic markers in sporadic amyotrophic lateral sclerosis. I Neuropathol Exp Neurol 66:617-27

Kaikkonen MU, Lam MT, Glass CK. 2011. Non-coding RNAs as regulators of gene expression and epigenetics. Cardiovasc Res 90:430-40

Kirby J, Halligan E, Baptista MJ, Allen S, Heath PR, et al. 2005. Mutant SOD1 alters the motor neuronal transcriptome: implications for familial ALS. Brain 128:1686-706

Kirby J, Ning K, Ferraiuolo L, Heath PR, Ismail A, et al. 2011. Phosphatase and tensin homologue/protein kinase B pathway linked to motor neuron survival in human superoxide dismutase 1-related amyotrophic lateral sclerosis. Brain 134:506-17

Lederer CW, Torrisi A, Pantelidou M, Santama N, Cavallaro S. 2007. Pathways and genes differentially expressed in the motor cortex of patients with sporadic amyotrophic lateral sclerosis. BMC Genomics 8:26

Lewis BP, Burge CB, Bartel DP. 2005. Conserved seed pairing, often flanked by adenosines, indicates that thousands of human genes are microRNA targets. Cell 120:15-20

Liew CC, Ma J, Tang HC, Zheng R, Dempsey AA. 2006. The peripheral blood transcriptome dynamically reflects system wide biology: a potential diagnostic tool. The Journal of Laboratory and Clinical Medicine 147:126-32

Liu J, Walter E, Stenger D, Thach D. 2006. Effects of globin mRNA reduction methods on gene expression profiles from whole blood. J Mol Diagn 8:551-8

Maes OC, Xu S, Yu B, Chertkow HM, Wang E, Schipper HM. 2007. Transcriptional profiling of Alzheimer blood mononuclear cells by microarray. Neurobiology of Aging 28:1795809

Malaspina A, Kaushik N, de Belleroche J. 2001. Differential expression of 14 genes in amyotrophic lateral sclerosis spinal cord detected using gridded cDNA arrays. $J$ Neurochem 77:132-45

Menzies FM, Cookson MR, Taylor RW, Turnbull DM, Chrzanowska-Lightowlers ZM, et al. 2002a. Mitochondrial dysfunction in a cell culture model of familial amyotrophic lateral sclerosis. Brain 125:1522-33

Menzies FM, Grierson AJ, Cookson MR, Heath PR, Tomkins J, et al. 2002b. Selective loss of neurofilament expression in $\mathrm{Cu} / \mathrm{Zn}$ superoxide dismutase (SOD1) linked amyotrophic lateral sclerosis. J Neurochem 82:1118-28

Mohr S, Liew CC. 2007. The peripheral-blood transcriptome: new insights into disease and risk assessment. Trends Mol Med 13:422-32

Mortiboys H, Thomas KJ, Koopman WJ, Klaffke S, Abou-Sleiman P, et al. 2008. Mitochondrial function and morphology are impaired in parkin-mutant fibroblasts. Ann Neurol 64:555-65

Nagai M, Re DB, Nagata T, Chalazonitis A, Jessell TM, et al. 2007. Astrocytes expressing ALS-linked mutated SOD1 release factors selectively toxic to motor neurons. Nat Neurosci 10:615-22 
Nagasaka Y, Dillner K, Ebise H, Teramoto R, Nakagawa H, et al. 2005. A unique gene expression signature discriminates familial Alzheimer's disease mutation carriers from their wild-type siblings. Proc Natl Acad Sci U S A 102:14854-9

Neymotin A, Calingasan NY, Wille E, Naseri N, Petri S, et al. 2011. Neuroprotective effect of Nrf2/ARE activators, CDDO ethylamide and CDDO trifluoroethylamide, in a mouse model of amyotrophic lateral sclerosis. Free Radic Biol Med 51:88-96

Offen D, Barhum Y, Melamed E, Embacher N, Schindler C, Ransmayr G. 2009. Spinal cord mRNA profile in patients with ALS: comparison with transgenic mice expressing the human SOD-1 mutant. J Mol Neurosci 38:85-93

Olsen MK, Roberds SL, Ellerbrock BR, Fleck TJ, McKinley DK, Gurney ME. 2001. Disease mechanisms revealed by transcription profiling in SOD1-G93A transgenic mouse spinal cord. Ann Neurol 50:730-40

Perrin FE, Boisset G, Docquier M, Schaad O, Descombes P, Kato AC. 2005. No widespread induction of cell death genes occurs in pure motoneurons in an amyotrophic lateral sclerosis mouse model. Hum Mol Genet 14:3309-20

Polymenidou M, Lagier-Tourenne C, Hutt KR, Huelga SC, Moran J, et al. 2011. Long premRNA depletion and RNA missplicing contribute to neuronal vulnerability from loss of TDP-43. Nat Neurosci 14:459-68

Ripps ME, Huntley GW, Hof PR, Morrison JH, Gordon JW. 1995. Transgenic mice expressing an altered murine superoxide dismutase gene provide an animal model of amyotrophic lateral sclerosis. Proc Natl Acad Sci U S A 92:689-93

Saris CG, Horvath S, van Vught PW, van Es MA, Blauw HM, et al. 2009. Weighted gene coexpression network analysis of the peripheral blood from Amyotrophic Lateral Sclerosis patients. BMC Genomics 10:405

Sarlette A, Krampfl K, Grothe C, Neuhoff N, Dengler R, Petri S. 2008. Nuclear erythroid 2related factor 2-antioxidative response element signaling pathway in motor cortex and spinal cord in amyotrophic lateral sclerosis. J Neuropathol Exp Neurol 67:1055-62

Scherzer CR, Eklund AC, Morse LJ, Liao Z, Locascio JJ, et al. 2007. Molecular markers of early Parkinson's disease based on gene expression in blood. Proc Natl Acad Sci U S A 104:955-60

Sharp FR, Lit L, Xu H, Apperson M, Walker W, et al. 2006. Genomics of brain and blood: progress and pitfalls. Epilepsia 47:1603-7

Shtilbans A, Choi SG, Fowkes ME, Khitrov G, Shahbazi M, et al. 2011. Differential gene expression in patients with amyotrophic lateral sclerosis. Amyotroph Lateral Scler 12:250-6

Silani V, Messina S, Poletti B, Morelli C, Doretti A, et al. 2011. The diagnosis of Amyotrophic lateral sclerosis in 2010. Arch Ital Biol 149:5-27

Son EY, Ichida JK, Wainger BJ, Toma JS, Rafuse VF, et al. 2011. Conversion of Mouse and Human Fibroblasts into Functional Spinal Motor Neurons. Cell Stem Cell DOI: 10.1016/j.stem.2011.07.014:(Epub ahead of print)

Stallings NR, Puttaparthi K, Luther CM, Burns DK, Elliott JL. 2010. Progressive motor weakness in transgenic mice expressing human TDP-43. Neurobiol Dis 40:404-14 
Stoughton RB. 2005. Applications of DNA microarrays in biology. Annu Rev Biochem 74:5382

Sultan M, Schulz MH, Richard H, Magen A, Klingenhoff A, et al. 2008. A global view of gene activity and alternative splicing by deep sequencing of the human transcriptome. Science 321:956-60

Sutherland GT, Janitz M, Kril JJ. 2011. Understanding the pathogenesis of Alzheimer's disease: will RNA-Seq realize the promise of transcriptomics? J Neurochem 116:93746

Tollervey JR, Curk T, Rogelj B, Briese M, Cereda M, et al. 2011. Characterizing the RNA targets and position-dependent splicing regulation by TDP-43. Nat Neurosci 14:4528

Tsuang MT, Nossova N, Yager T, Tsuang MM, Guo SC, et al. 2005. Assessing the validity of blood-based gene expression profiles for the classification of schizophrenia and bipolar disorder: a preliminary report. Am J Med Genet B Neuropsychiatr Genet 133B:1-5

Turner MR, Kiernan MC, Leigh PN, Talbot K. 2009. Biomarkers in amyotrophic lateral sclerosis. Lancet Neurol 8:94-109

Vargas MR, Johnson DA, Sirkis DW, Messing A, Johnson JA. 2008a. Nrf2 activation in astrocytes protects against neurodegeneration in mouse models of familial amyotrophic lateral sclerosis. J Neurosci 28:13574-81

Vargas MR, Pehar M, Cassina P, Beckman JS, Barbeito L. 2006. Increased glutathione biosynthesis by Nrf2 activation in astrocytes prevents p75NTR-dependent motor neuron apoptosis. J Neurochem 97:687-96

Vargas MR, Pehar M, Diaz-Amarilla PJ, Beckman JS, Barbeito L. 2008b. Transcriptional profile of primary astrocytes expressing ALS-linked mutant SOD1. J Neurosci Res $86: 3515-25$

Wang XS, Simmons Z, Liu W, Boyer PJ, Connor JR. 2006. Differential expression of genes in amyotrophic lateral sclerosis revealed by profiling the post mortem cortex. Amyotroph Lateral Scler 7:201-10

Wang Z, Gerstein M, Snyder M. 2009. RNA-Seq: a revolutionary tool for transcriptomics. Nat Rev Genet 10:57-63

Whitney AR, Diehn M, Popper SJ, Alizadeh AA, Boldrick JC, et al. 2003. Individuality and variation in gene expression patterns in human blood. Proc Natl Acad Sci U S A 100:1896-901

Williams AH, Valdez G, Moresi V, Qi X, McAnally J, et al. 2009. MicroRNA-206 delays ALS progression and promotes regeneration of neuromuscular synapses in mice. Science 326:1549-54

Wright C, Bergstrom D, Dai H, Marton M, Morris M, et al. 2008. Characterization of globin RNA interference in gene expression profiling of whole-blood samples. Clinical chemistry 54:396-405

Yoshihara T, Ishigaki S, Yamamoto M, Liang Y, Niwa J, et al. 2002. Differential expression of inflammation- and apoptosis-related genes in spinal cords of a mutant SOD1 transgenic mouse model of familial amyotrophic lateral sclerosis. J Neurochem 80:158-67 
Zhang R, Hadlock KG, Do H, Yu S, Honrada R, et al. 2011. Gene expression profiling in peripheral blood mononuclear cells from patients with sporadic amyotrophic lateral sclerosis (sALS). J Neuroimmunol 230:114-23

Zoccolella S, Beghi E, Palagano G, Fraddosio A, Samarelli V, et al. 2006. Predictors of delay in the diagnosis and clinical trial entry of amyotrophic lateral sclerosis patients: a population-based study. J Neurol Sci 250:45-9 
AMYOTROPHIC

LATERAL SCLEROSIS

Eaced by Marton H. maver

\section{Amyotrophic Lateral Sclerosis}

Edited by Prof. Martin Maurer
ISBN 978-953-307-806-9

Hard cover, 718 pages

Publisher InTech

Published online 20, January, 2012

Published in print edition January, 2012

Though considerable amount of research, both pre-clinical and clinical, has been conducted during recent years, Amyotrophic Lateral Sclerosis (ALS) remains one of the mysterious diseases of the 21 st century. Great efforts have been made to develop pathophysiological models and to clarify the underlying pathology, and with novel instruments in genetics and transgenic techniques, the aim for finding a durable cure comes into scope. On the other hand, most pharmacological trials failed to show a benefit for ALS patients. In this book, the reader will find a compilation of state-of-the-art reviews about the etiology, epidemiology, and pathophysiology of ALS, the molecular basis of disease progression and clinical manifestations, the genetics familial ALS, as well as novel diagnostic criteria in the field of electrophysiology. An overview over all relevant pharmacological trials in ALS patients is also included, while the book concludes with a discussion on current advances and future trends in ALS research.

\section{How to reference}

In order to correctly reference this scholarly work, feel free to copy and paste the following:

Johnathan Cooper-Knock, Joanna J. Bury, Laura Ferraiuolo, Emily F. Goodall, Pamela J. Shaw and Janine Kirby (2012). Insights Arising from Gene Expression Profiling in Amyotrophic Lateral Sclerosis, Amyotrophic Lateral Sclerosis, Prof. Martin Maurer (Ed.), ISBN: 978-953-307-806-9, InTech, Available from: http://www.intechopen.com/books/amyotrophic-lateral-sclerosis/insights-arising-from-gene-expressionprofiling-in-amyotrophic-lateral-sclerosis

\section{INTECH}

open science | open minds

\section{InTech Europe}

University Campus STeP Ri

Slavka Krautzeka 83/A

51000 Rijeka, Croatia

Phone: +385 (51) 770447

Fax: +385 (51) 686166

www.intechopen.com

\section{InTech China}

Unit 405, Office Block, Hotel Equatorial Shanghai

No.65, Yan An Road (West), Shanghai, 200040, China

中国上海市延安西路65号上海国际贵都大饭店办公楼405单元

Phone: +86-21-62489820

Fax: $+86-21-62489821$ 
(C) 2012 The Author(s). Licensee IntechOpen. This is an open access article distributed under the terms of the Creative Commons Attribution 3.0 License, which permits unrestricted use, distribution, and reproduction in any medium, provided the original work is properly cited. 